\title{
Caracterización de materia prima utilizada para fabricación artesanal de unidades de mampostería en las ladrilleras El Cielo y Las Casitas, Valledupar - Colombia
}

\section{Characterizing Raw Material Used for Artisanal Production of Masonry Units in Brickyards El Cielo and Las Casitas, Valledupar, Colombia}

Recibido: 04 de septiembre de 2020. Recibido en revisión: 13 de octubre de 2020.

Aceptado: 03 de noviembre de 2020. DOl: https://doi.org/10.33132/27114260.1908 Galindo González, José C4; Crespo Guerra, María Teresa ${ }^{5}$; Mahecha Meza, Oscar Antonio Rivero Galvis, Naty Vanesa ; Orozco Ospino, Javier Enrique ${ }^{8}$; García-León, Ricardo Andrés ${ }^{9}$

\section{Resumen}

Estudios conducidos en años recientes han concentrado su interés en incorporar materiales no convencionales para fabricar unidades de mampostería. Esto no ha sido

\footnotetext{
4 Facultad de Ingeniería y Ciencias Básicas, Fundación Universitaria del Área Andina, Valledupar, Colombia. Grupo de investigación e innovación en ingeniería civil (GINC), jgalindo222areandina.edu.co, https://orcid.org/0000-0002-4810-0866 ,jaorozcođareandina.edu.co, https://orcid.org/0000-0002-4234-7630

5.Facultad de Ingeniería y Ciencias Básicas, Programa Ingeniería Civil, Fundación Universitaria del Área Andina, Valledupar, Colombia.mcrespodestudiantes.areandina.edu.co, omahechađestudiantes.areandina.edu.co), nrivero4(dareandina.edu.co.

6 Facultad de Ingeniería y Ciencias Básicas, Programa Ingeniería Civil, Fundación Universitaria del Área Andina, Valledupar, Colombia.mcrespodestudiantes.areandina.edu.co, omahechađestudiantes.areandina.edu.co), nrivero4(aareandina.edu.co.

7 Facultad de Ingeniería y Ciencias Básicas, Programa Ingeniería Civil, Fundación Universitaria del Área Andina, Valledupar, Colombia.mcrespo(destudiantes.areandina.edu.co, omahecha(destudiantes.areandina.edu.co), nrivero4dareandina.edu.co.

8 Facultad de Ingeniería y Ciencias Básicas, Fundación Universitaria del Área Andina, Valledupar, Colombia. Grupo de investigación e innovación en ingeniería civil (GINC), jgalindo22Rareandina.edu.co, https://orcid.org/0000-0002-4810-0866 ,jaorozcođareandina.edu.co, https://orcid.org/0000-0002-4234-7630

9 Facultad de Ingenierías, Universidad Francisco de Paula Santander, Ocaña, Colombia Grupo de investigación en ingenierías aplicadas para la innovación, gestión y desarrollo (INGAP), ragarcialdaufpso.edu.co https://orcid.org/0000-0002-2734-1425
} 
óbice para continuar examinando materias primas tradicionales. El trabajo consistió en caracterizar dos materiales explotados para producción artesanal de unidades macizas de mampostería. Las ladrilleras se localizan al sur del área urbana de Valledupar, Colombia. Muestras representativas del material se tomaron de vetas explotadas por cada fabricante a los que se les practicaron ensayos que permitieron clasificarlos y determinar su composición química y mineralógica, al tiempo que se determinó la resistencia mecánica de productos terminados. Las composiciones granulométricas indican predominancia de limos en El Cielo y de arena en Las Casitas. Ambos materiales son aptos para fabricar ladrillos perforados en lugar de macizos. Los índices de plasticidad y limite plástico en ambos casos sustentan irregularidades observadas en aristas de unidades terminadas, denotando dificultad en desmoldado. La resistencia promedio a compresión obtenida de ladrillos terminados indica que no son aptos para mampostería.

Palabras clave: arcilla, ladrillo, mampostería, materiales de construcción.

\section{Abstract}

Studies conducted in recent years have focused their interest on incorporating unconventional materials to manufacture masonry units, which has not been an obstacle to continue examining traditional raw materials. This paper is about characterizing two materials exploited for the artisanal production of solid masonry units. The brickyards are located south of the urban area of Valledupar, Colombia. Representative samples of the material were taken from veins exploited by each manufacturer, performing tests to classify them and determine their chemical and mineralogical composition while defining the mechanical resistance of finished products. The granulometric compositions indicate a predominance of silt in El Cielo and sand in Las Casitas; both materials are suitable for making perforated bricks rather than solid bricks. In both cases, the plasticity and plastic limit indices support irregularities observed in the edges of finished units, denoting difficulty in demolding. The average compressive strength obtained from finished bricks suggests that they are unsuitable for masonry.

Keywords: Clay, brick, masonry, building materials. 


\section{Introducción}

En los años recientes se han incrementado los planteamientos en diversos sectores que pretenden incorporar materiales no convencionales para la generación de productos utilizados en la industria de la construcción (Al-Fakih et al., 2019; Ghavami, 2020; Phonphuak y Chindaprasirt, 2015). Muchos de esos nuevos materiales son subproductos derivados de los desechos que la sociedad de consumo genera luego de un primer uso (Al-Fakih et al., 2019). Al tiempo que esto sucede, en muchos países en vía de desarrollo se siguen estudiando materias primas con las que se manufacturan a menor escala productos que cubren la demanda de materiales de construcción en mercados locales (Suffian et al., 2020). Algunos trabajos de referencia dan cuenta de la necesidad de conocer aspectos y características de las arcillas con las que se producen los materiales cerámicos que abastecen la industria, proponiendo establecer aquellos cuya mayor relevancia son asociados con la calidad (Amado et al., 2011; Flórez Arenas, 2016; García-León, Flórez Solano et al., 2018; García León y Bolívar León, 2017).

El estudio busca conocer en detalle aspectos técnicos específicos de la ma- teria prima empleada por dos ladrilleras, quienes la utilizan para manufactura de unidades macizas que se comercializan en el mercado local de materiales de construcción. Se obtuvieron muestras de los materiales que explota cada ladrillera y se realizaron ensayos para determinar su composición granulométrica, química y mineralógica, además de los límites de consistencia. Los resultados obtenidos muestran la aptitud de estos materiales para fabricar productos cerámicos.

\section{Materiales y metodología}

Dentro del planteamiento descriptivo de este trabajo se realizó inspección de campo en los sitios donde cada productor desarrollaba la manufactura de ladrillos. Posteriormente se realizó una jornada de muestreo en la cual se obtuvieron tres (3) muestras representativas del material que utilizaba cada productor, dos para observación y una tercera para control. Las de El Cielo fueron rotuladas como EC1, EC2 y EC3. En el caso de Las Casitas fueron LK1, LK2 y LK3. Del material explotado en cada cantera se obtuvieron muestras inalteradas del suelo de 50 kilogramos cada una, aproximadamente. A estas muestras se les aplicaron los ensayos que se relacionan en la tabla 1. También se escogieron aleatoriamente cinco (5) muestras de 
Tabla 1. Ensayos realizados.

\begin{tabular}{|c|c|c|}
\hline PROPÓSITO & ENSAYO & NORMA \\
\hline $\begin{array}{l}\text { Obtención de muestras representati- } \\
\text { vas para ensayos }\end{array}$ & $\begin{array}{l}\text { Selección muestra para ensayos de granu- } \\
\text { lometría por método de cuarteo manual y } \\
\text { preparación muestras por vía seca }\end{array}$ & $\begin{array}{l}\text { INV E - } 202-13 \\
\text { ASTM C702/C702M - } 11 \\
\text { INV E - } 106-13 \\
\text { ASTM D421 - } 85\end{array}$ \\
\hline \multirow{3}{*}{$\begin{array}{l}\text { Composición y clasificación del } \\
\text { material }\end{array}$} & $\begin{array}{l}\text { Análisis granulométrico por tamizado y por } \\
\text { hidrómetro }\end{array}$ & $\begin{array}{l}\text { INV E - } 123 \text { - 13, NTC 4630, ASTM } \\
\text { D422 - } 63\end{array}$ \\
\hline & Limite líquido, LL & $\begin{array}{l}\text { INV E - } 125 \text { - 13, NTC 4630, ASTM } \\
\text { D4318 - } 17\end{array}$ \\
\hline & $\begin{array}{l}\text { Limite plástico, LP } \\
\text { Îndice de plasticidad, IP }\end{array}$ & $\begin{array}{l}\text { INV E - } 126-13, \text { NTC 4630, ASTM } \\
\text { D4318 - } 17\end{array}$ \\
\hline Caracterización química & Fluorescencia o espectrografía de rayos X & ASTM C323 - 56 \\
\hline Caracterización mineralógica & Difracción de rayos $X$ & ASTM E975 - 13 \\
\hline $\begin{array}{l}\text { Resistencia mecánica a especímenes } \\
\text { terminados }\end{array}$ & Ensayo a compresión & $\begin{array}{l}\text { NTC 4017, ASTM C67/C67M - } 20 \text {, } \\
\text { NTC 4205-2, ASTM C56 - } 13\end{array}$ \\
\hline
\end{tabular}

Fuente: elaboración propia.

ladrillos del último lote que disponía cada fabricante en el sitio, los cuales fueron producidos a partir del mismo material llevado al laboratorio.

Del proceso de cuarteo para las muestras de control (EC3 y LK3) se obtuvo una muestra representativa de cada cantera a las que se le realizaron las pruebas de espectrografía de rayos $\mathrm{X}$ (FRX) y de difracción de rayos X (DRX). Para la de FRX se utilizó el equipo S4 Explorer de Bruker, con detector Pro4, tubo de ensayo $\mathrm{X} \mathrm{Rh}$ a un voltaje de $40 \mathrm{kV}$ a 25 mA. Para la de DRX se utilizó el equipo D4 AXS de Bruker, con detector LYNXEYE, Tubo de rayos-X Cu $1.9 \mathrm{Kw}$
( $\mathrm{K}$ alpha $\lambda=1.5406$ ) con filtro de níquel a $40 \mathrm{KV} 40 \mathrm{~mA}$, con un haz de electrones normales y ángulos de incidencia 20: 5 $70^{\circ}$ (montaje polvo), 5 - $35^{\circ}$ (agregados orientados) y una velocidad de $0.4 \mathrm{~s} / \theta$.

\section{Resultados y discusión}

Los datos que se derivan de los ensayos practicados a las muestras se exponen a continuación.

Análisis granulométrico por tamizado. Se obtuvo la composición de la muestra de material cuya fracción queda retenida en el tamiz No. 10 (tamaños mayores a 2.0 milímetros). Los porcentajes que se retuvieron en dicho tamiz 
para las muestras EC1, EC2 y EC3 fueron $0.0 \%, 0.4 \%$ y $0.3 \%$. En el caso de las muestras LK1, LK2 y LK3 fueron $2.8 \%, 1.7 \%$ y $0.5 \%$. La representación de curvas granulometrías de las muestras no son relevantes para el estudio dado que están conformados mayoritariamente por finos.

Análisis granulométrico por método del hidrómetro. Con este método se obtuvo la composición de la muestra cuya fracción pasa el tamiz No. 10 (tamaños menores a 2.0 milímetros). La siguiente relación muestra la composición, expresada como porcentaje, de arcilla, limo y arena obtenido para cada muestra.
En la composición obtenida para las muestras de El Cielo se observó predominancia de limo. En el caso de Las Casitas se observó predominancia de arena.

Limites de consistencia. La tabla 3 presenta los valores obtenidos de los límites de Atterberg de las muestras. Con estos se clasifica la muestra de suelo y se establece el grupo al que pertenece. En el sistema unificado de clasificación de suelos (SUCS) la muestra de El Cielo arrojó dos limos y una arcilla de baja compresibilidad; en la de Las Casitas se obtuvieron arcillas limo arenosas.

Tabla 2. Composición muestras fracción que pasa el tamiz №. 10.

\begin{tabular}{|c|c|c|c|c|}
\hline \multirow[t]{2}{*}{ Productor } & \multirow[t]{2}{*}{ Muestra } & $\begin{array}{l}\text { Arcilla } \\
<2 \mu \mathrm{m}\end{array}$ & $\begin{array}{l}\text { Arena } \\
2 \text { a } 20 \mu \mathrm{m}\end{array}$ & $\begin{array}{l}\operatorname{Lim0} \\
>20 \mu \mathrm{m}\end{array}$ \\
\hline & & \multicolumn{3}{|c|}{$\%$} \\
\hline \multirow{3}{*}{ El Cielo } & EC1 & 21.16 & 17.20 & 61.64 \\
\hline & $\mathrm{EC2}$ & 34.64 & 20.70 & 44.66 \\
\hline & EC3 & 24.85 & 35.60 & 39.55 \\
\hline \multirow{3}{*}{ Las Casitas } & LK1 & 37.40 & 54.00 & 8.60 \\
\hline & LK2 & 26.00 & 55.50 & 18.50 \\
\hline & LK3 & 25.44 & 41.20 & 33.36 \\
\hline
\end{tabular}

Fuente: elaboración propia. 
Tabla 3. Límites de consistencia muestras.

\begin{tabular}{lllll}
\hline Productor & Muestra & Límite Líquido, LL & $\begin{array}{l}\text { Límite Plástico, } \\
\text { LP }\end{array}$ & $\begin{array}{l}\text { Índice de } \\
\text { Plasticidad, } \\
\text { IP }\end{array}$ \\
\hline \multirow{3}{*}{ El Cielo } & EC1 & 30.9 & & \\
\hline \multirow{3}{*}{ Las Casitas } & EC2 & 34.9 & 23.4 & 7.51 \\
& EC3 & 31.0 & 25.4 & 9.43 \\
& LK1 & 31.5 & 21.4 & 9.60 \\
\hline & LK2 & 22.0 & 19.9 & 11.56 \\
\hline
\end{tabular}

Fuente: Elaboración propia.

\section{Aptitud del material para fabricación de unidades de mampostería}

Aplicando el tratamiento dado por algunos autores a los datos de composición de los finos se utiliza el software Triplop, con el cual se grafica en el diagrama de Winkler y se realiza, además, un análisis estadístico de primer orden para los datos graficados (García-León, Flórez Solano, et al., 2018; García León y Bolívar León, 2017). Luego del procesamiento de datos y con el análisis precitado, se obtienen los siguientes resultados para las muestras de cada productor, las cuales se supone se ajustan a una distribución normal.
Derivado de la representación en el diagrama de Winkler para el caso de las texturas se obtuvo que las muestras de El Cielo fueron franca limosa, franca arcillosa y franca, para EC1, EC2 y EC3, respectivamente. Las observadas para las muestras de Las Casitas fueron arcilla arenosa, franca arcillosa arenosa y franca para LK1, LK2 y LK3, respectivamente. Los coeficientes de variación obtenidos para los componentes del material fino de las muestras analizadas dan cuenta de la naturaleza heterogénea del material de estudio, característica inherente de los suelos. 
Tabla 4. Resumen composición granulométrica por hidrómetro.

\begin{tabular}{lllllll} 
& \multicolumn{3}{c}{ Muestras El Cielo } & \multicolumn{5}{c}{ Muestras Las Casitas } \\
& Arcilla & Arena & Limo & Arcilla & Arena & Limo \\
\hline Composición promedio (\%) & 26.88 & 24.50 & 48.62 & 29.61 & 50.23 & 20.15 \\
Desviación estándar (\%) & 7.0 & 9.8 & 11.6 & 6.7 & 7.9 & 6.5 \\
Coeficiente de variación (\%) & 25.9 & 39.9 & 23.8 & 22.8 & 15.6 & 61.8 \\
\hline
\end{tabular}

Fuente: elaboración propia.

Respecto a la capacidad del material para manufactura de unidades de mampostería, ambos materiales muestran una aptitud predominante para que se utilicen en la producción de unidades

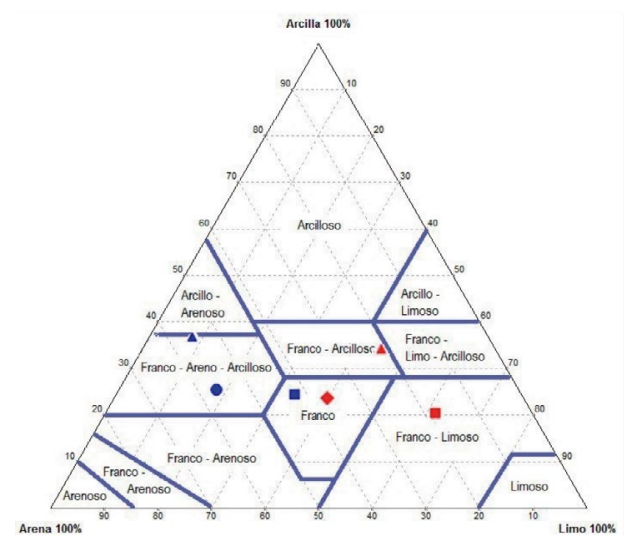

(a) Representación textura perforadas. Los datos graficados en el diagrama de Winkler resultan de utilidad tal como lo han expuesto trabajos previos (García-León, Flórez Solano, et al., 2018; Mendonça et al., 2017), pues-

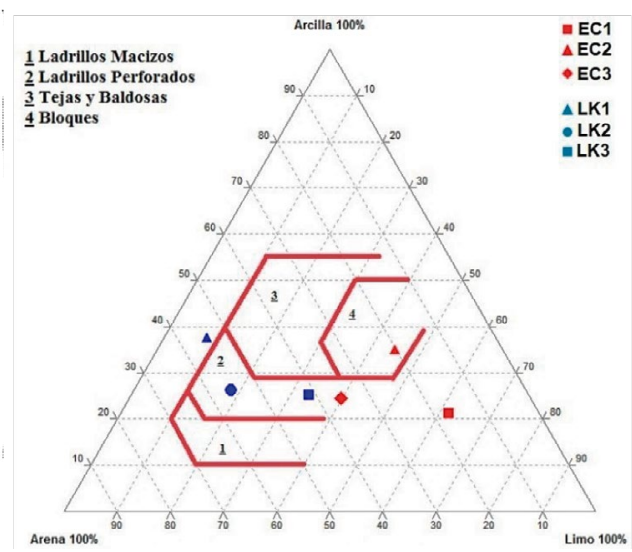

(b) Representación aptitud para

fabricar productos cerámicos

Figura 1. Representación composición muestras en diagrama de Winkler.

Fuente: elaboración propia. 
to que permiten determinar, a través de la dosificación de alguno de los componentes las cuantías, el material que debe adicionarse para mejorar la aptitud en la manufactura de alguno de los elementos indicados en la figura 1(b).

Otra particularidad que denota la aptitud del material para usarse en la manufactura de productos cerámicos se obtiene a través de los límites de consistencia. En este caso se graficaron en el diagrama de Casagrande IP y LP de las muestras. A partir de este arreglo se obtuvo la noción del grado de dificultad para desmoldar los especímenes en el momento de su producción. Asociado a esto, las irregularidades observadas en las aristas de unidades terminadas bien pudieron derivarse de un proceso de desmoldado entre difícil y aceptable, tal como se predice y evidencia en las figuras 2 (a) y 2 (b).

\section{Composición química}

La composición obtenida mediante espectrografía de rayos $\mathrm{X}$ permitió determinar los componentes químicos presentes en la muestra de cada cantera. Se obtuvieron para las muestras can- tidad mayoritaria de óxidos de Silicio $\left(\mathrm{SiO}_{2}\right)$, de Aluminio $\left(\mathrm{Al}_{2} \mathrm{O}_{3}\right)$, de Hierro $\left(\mathrm{Fe}_{2} \mathrm{O}_{3}\right)$ y de Calcio $(\mathrm{CaO})$, en los siguientes porcentajes para la muestra de El Cielo - 64.01\%, $15.98 \%, 6.25 \%$ y $2.62 \%$, respectivamente-; y en la de Las Casitas —61.07 \%, $16.69 \%, 7.29$ $\%$ y $2.50 \%$, respectivamente-. En los trabajos de Flórez Arenas (2016), Mahmoudi et al., (2017) y García-León, Flórez-Solano, et al., (2018) se reseña que los materiales se consideran aptos para manufactura de elementos cerámicos si en su composición se registra de $50 \%$ a $60 \%$ de óxido de silicio y entre $20 \%$ a $30 \%$ de óxido de aluminio. En este caso, se observó que los materiales de ambas canteras se encuentran por fuera de dichos rangos. En el mismo sentido Amado et al. (2011) plantean que composiciones de $\mathrm{A} 12 \mathrm{O} 3$ cercanas a $30 \%$ permiten elaborar ladrillos refractarios. Esto indica que, aunque se someten a un proceso de cocción, los especímenes producidos con el material de las muestras ensayadas no desarrollan resistencias mecánicas para clasificar en el apartado de productos conforme a las normas aplicables. 


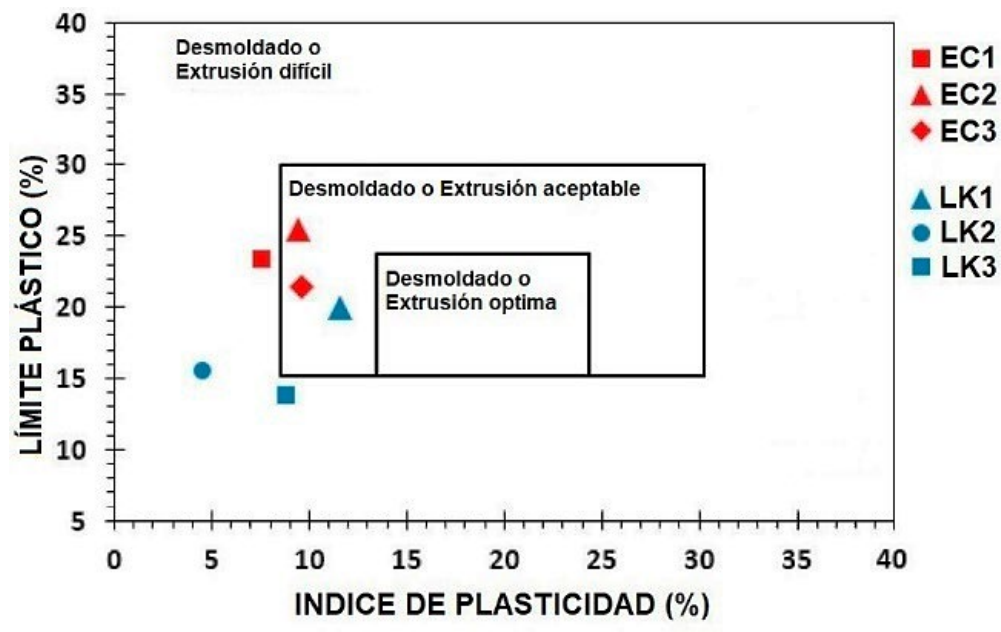

(a) Predicción desmoldado de muestras basados en los límites de consistencia

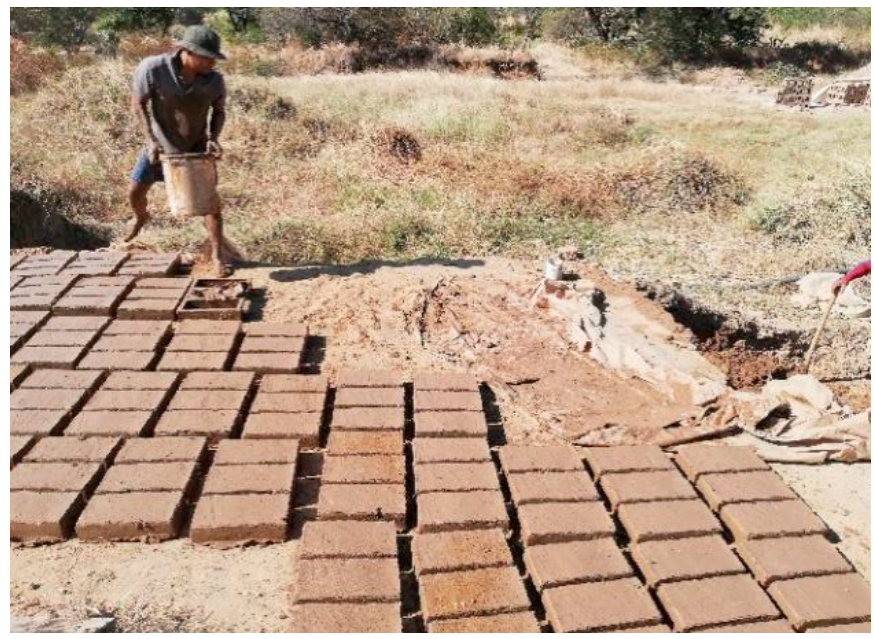

(b) Muestras posterior al desmoldado, antes del proceso de cocción

Figura 2. Aspectos relacionados con desmoldado o extrusión de muestras.

Fuente: elaboración propia. 


\section{Composición mineralógica}

Las figuras 3(a) y 3(b) muestran difractogramas de las muestras a las que se les aplicaron este ensayo en particular. Para las fases cristalinas se observaron mayoritariamente cuarzo libre, albita libre, microclina y muscovita, de las cuales se reportó en El Cielo $41.0 \%, 32.8 \%, 20.2 \%$ y $4.8 \%$ y $43.5 \%, 30.5 \%, 14.0 \%$ y $9.8 \%$, respectivamente, en Las Casitas.

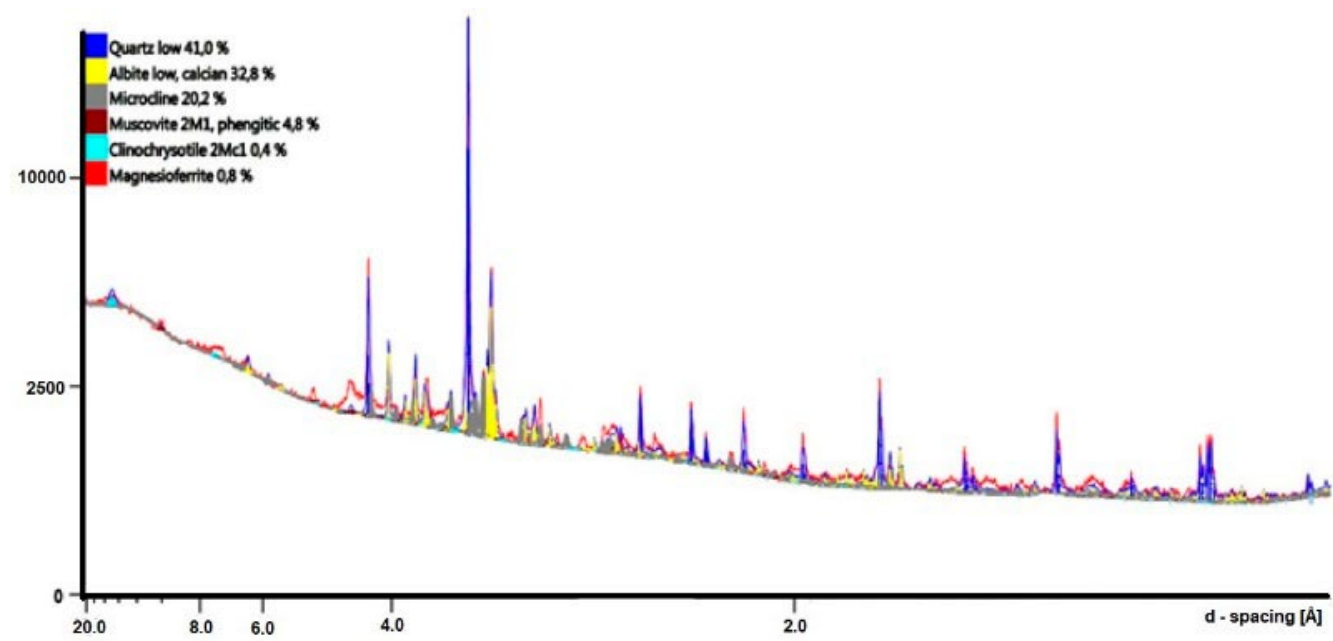

(a) Muestra EC3

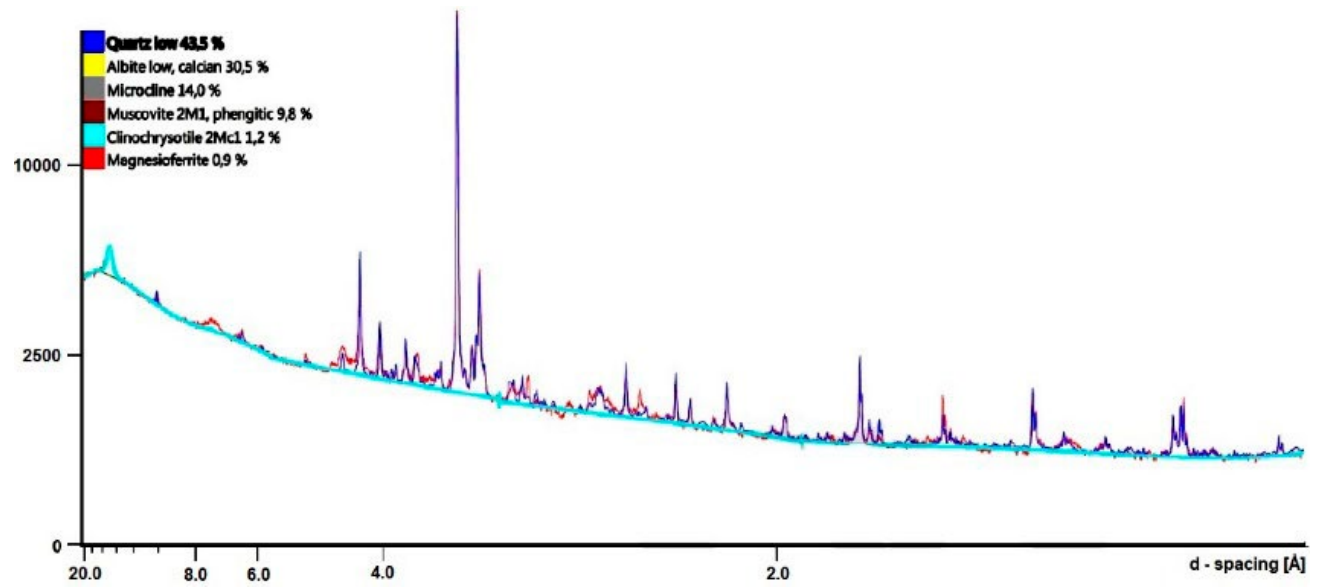

(b) Muestra LK3

Figura 3. Difractograma de rayos $X$.

Fuente: elaboración propia. 
Los niveles de cuarzo observados indican la propiedad desgrasante del material, lo que evita que en el amasado se adquiera una plasticidad excesiva de la mezcla disminuyendo la posibilidad de que ocurran contracciones y el subsecuente agrietamiento de esta durante el secado y la cocción. La presencia de feldespatos denotada por la albita y microclima indica que se puede fijar el color característico y homogéneo blanquecino a productos producidos con dichos materiales, además que actúa como fundente en la cocción. La moscovita en menor proporción denota mediana capacidad de resistir al proceso de cocción sin que se afecte la estabilidad dimensional de los especímenes. Algunas particularidades observadas en arcillas utilizadas para producción de elementos cerámicos han sido reportadas en prospecciones realizadas por varios autores (Amado $e t$ al., 2011; Florez Arenas, 2016; GarcíaLeón, Flórez-Solano, et al., 2018; Mahmoudi et al., 2017).

\section{Ensayo a compresión unidades terminadas}

Registros de las cinco unidades ensayadas de cada productor se promediaron. Para el caso de El Cielo se obtuvo 8.8 $\pm 2.2 \mathrm{MPa}$ y para Las Casitas $9.2 \pm 0.8$
MPa. Al comparar ambos resultados con lo establecido en la norma de referencia aplicable para unidades de mampostería no estructural, NTC 4205-2, ambas están por debajo del mínimo estandarizado de $14 \mathrm{MPa}$. Comparando estos datos con los de resistencia reportados en el trabajo de Cotes et al., (2012), los del presente estudio denotan un incremento de resistencia alcanzada para las unidades de mampostería que se producen en la zona.

\section{Conclusiones}

Con los datos de composición granulométrica se establecieron las características que indican la aptitud del material para producir unidades cerámicas diferentes a las que se producen actualmente por métodos artesanales. Los límites de consistencia permitieron predecir que el desmoldado en el proceso de elaboración de los especímenes puede tener incidencia en las irregularidades observadas en las aristas de unidades terminadas. Derivados de la composición química y mineralógica obtenidas en el reporte de las muestras ensayadas, se deducen aspectos de relevancia que son indicativos de la calidad del producto manufacturado por ambas ladrilleras. Es razonable concluir que los valores de las resistencias observadas de las unidades terminadas corroboran lo establecido en la literatura 
de referencia, pues para que un material se denote como refractario requiere que se registren en su estructura química algunos compuestos específicos que en las muestras se detectaron en proporciones por fuera de los rangos indicados.

\section{Agradecimientos}

Los autores expresan sus agradecimientos al ingeniero Alais Rojas Montero y al laboratorio de ensayos PENDING SAS por los recursos facilitados en la realización de este trabajo.

\section{Referencias}

Al-Fakih, A., Mohammed, B. S., Liew, M. S., \& Nikbakht, E. (2019). Incorporation of waste materials in the manufacture of masonry bricks: An update review. Journal of Building Engineering, 21, 37-54. https:// doi.org/10.1016/j.jobe.2018.09.023

Amado, J. D. S., Villafrades, P. Y. M., \& Tuta, E. M. C. (2011). Caracterización de arcillas y preparación de pastas cerámicas para la fabricación de tejas y ladrillos en la región de Barichara, Santander. DYNA (Colombia), 78(167), 50-58. https://revistas.unal.edu.co/index.php/dyna/article/ view/25762/39346

ASTM D421 - 85 Standard Practice for Dry Preparation of Soil Samples for Particle-Size Analysis and Determination of Soil Constants, 2 (2007).
ASTM D422 - 63 Standard Test Method for Particle-Size Analysis of Soils, 8 (2007).

ASTM C702/C702M - 11 Standard Practice for Reducing Samples of Aggregate to Testing Size, 5 (2011).

ASTM E975 - 13 Standard Practice for X-Ray Determination of Retained Austenite in Steel with Near Random Crystallographic Orientation, 7 (2013).

ASTM C323 - 56 Standard Test Methods for Chemical Analysis of Ceramic Whiteware Clays, 5 (2016).

ASTM C56 - 13 Standard Specification for Structural Clay Nonloadbearing Tile, 3 (2017).

ASTM D4318 - 17 Standard Test Methods for Liquid Limit, Plastic Limit, and Plasticity Index of Soils, 20 (2017).

1ASTM C67/C67M - 20 Standard Test Methods for Sampling and Testing Brick and Structural Clay Tile, 17 (2020).

Cotes, D., Núñez, D., \& Sabogal, L. (2012). Determinación de la calidad de los ladrillos a partir de la estimación de la resistencia última en las canteras de Valencia de Jesús, Las Casitas y El Cielo, en el municipio de Valledupar, Cesar. Revista Agunkuyâa, 2(1), 32-41. https://revia.areandina.edu.co/ index.php/Cc/article/view/301

Florez Arenas, A. (2016). Caracterización de arcillas y preparación de pastas cerámicas para la fabricación de ladrillos en la ladrillera Mariscal Robledo S.A. Facultad de Tecnologías: Química Industrial, Universidad Tecnológica de Pereira, Colombia. http://hdl.handle.net/11059/6338 
1García-León, R. A., Flórez-Solano, E. N., \& Acevedo-Peñaloza, C. H. (2018). Clay surface characteristics using atomic force microscopy. Revista Facultad de Ingeniería, Universidad de Antioquia, 87, 23-24. https://doi.org/10.17533/udea.redin.n87a04

García-León, R. A., Flórez Solano, E., \& Rodríguez Castilla, M. (2018). Diseño de Mezclas para la Fabricación de Productos de Mampostería en la Industria Cerámica. Revista Politécnica, 14(26), 19-28. https:// doi.org/10.33571/rpolitec.v14n26a2

García León, R. A., \& Bolívar León, R. (2017). Caracterización hidrométrica de las arcillas utilizadas en la fabricación de productos cerámicos en Ocaña, Norte de Santander. INGE CUC, 13(1), 53-60. https://doi. org/10.17981/ingecuc.13.1.2017.05

Ghavami, K. (2020). Introduction to nonconventional materials and an historic retrospective of the field. In Nonconventional and Vernacular Construction Materials (2nd ed., pp. 37-61). Elsevier. https://doi. org/10.1016/B978-0-08-102704-2.00002-0

Norma Técnica Colombiana NTC 4630 Método de ensayo para la determinación del límite líquido, del límite plástico y del índice de plasticidad de los suelos cohesivos, 21 (1999).

Norma Técnica Colombiana NTC 4205-2 Unidades de mampostería de arcilla cocida. Ladrillos y bloques cerámicos. Parte 2: mampostería no estructural, 11 (2009).
Norma Técnica Colombiana NTC 4017 Métodos para muestreo y ensayos de unidades de mampostería y otros productos de arci1la, 50 (2018).

INV E - 106 - 13. Preparación en seco de muestras de suelo por vía seca para análisis granulométrico y determinación de las contantes físicas, Especificaciones generales de construcción de carreteras y normas de ensayo para materiales de carreteras (2013).

INV E - 123 - 13. Determinación de los tamaños de las partículas de los suelos, Especificaciones generales de construcción de carreteras y normas de ensayo para materiales de carreteras (2013).

INV E - 125 - 13. Determinación del límite líquido de los suelos, Especificaciones generales de construcción de carreteras y normas de ensayo para materiales de carreteras (2013).

INV E - 126 - 13. Limite plástico e índice de plasticidad de los suelos, Especificaciones generales de construcción de carreteras y normas de ensayo para materiales de carreteras (2013).

INV E - 202 - 13. Reducción de muestras de agregados por cuarteo, Especificaciones generales de construcción de carreteras y normas de ensayo para materiales de carreteras (2013).

Mahmoudi, S., Srasra, E., \& Zargouni, F. (2017). Preparation, qualities and defects of ceramic materials from Tunisian clay 
minerals. Surface Engineering and Applied Electrochemistry, 53(3), 295-301. https:// doi.org/10.3103/S1068375517030103

Mendonça, G. C., Ferreira, C. C., Fonseca, J. M., \& Quintana, L. H. (2017). Uso do diagrama de winkler para determinação da granulometria de uma argila in natura mais adições de cinzas residuais de carvão mineral. Anais Do V Congresso Brasileiro de Carvão Mineral. https://doi.org/10.20906/ cps/cbcm2017-0024

Phonphuak, N., \& Chindaprasirt, P. (2015). Types of waste, properties, and durability of pore-forming waste-based fired masonry bricks. In Eco-Efficient Masonry Bricks and Blocks (pp. 103-127). Elsevier. https://doi. org/10.1016/B978-1-78242-305-8.00006-1

28 Suffian, S., Dzombak, R., \& Mehta, K. (2020). Future directions for nonconventional and vernacular material research and applications. In Nonconventional and Vernacular Construction Materials (2nd ed., pp. 63-80). Elsevier. https://doi. org/10.1016/B978-0-08-102704-2.00003-2 